\title{
DSP BASED PLATFORM FOR AN ENHANCED HF CHANNEL SIMULATOR
}

\author{
R. Valle-Alarcón, Joan Serrat Fernández \\ Universitat Politecnica de Catalunya, Spain
}

\begin{abstract}
Whith the aim of improving existing tools for testing new HF transmission systems, this contribution is focussed on describing a modification of the basic Watterson model which can be implemented without any extra added complexity with a general purpuse DSP card running an a PC IBM compatible.
\end{abstract}

\section{INTRODUCTION}

The design of modern HF communication systems requires the ability to test the equipment in a much real conditions as possible. Testing in real life environments are often discarted due to the associated problems. Therefore the availability of channels simulators following closely the real propagation phenomena are mandatory.

HF channel simulators based on the Watterson model [1] have been for a long time a paradigm in the field. But what this model has as main drawback is that it doesn't allow any frequency variation, and the time variability is restricted to a very well defined statistical model. This is why the validity of the Watterson model, and indeed the simulator, is only for a few miliseconds and for narrowband signals. But today's HF transmission systems make use in more or less depth of real time channel evaluation techniques to exploit both the time and the frequency dependencies of the lonosphere. Therefore to test these systems the old model is no longer valid.

Besides other approaches, it seems reasonable that one way to reach the objective of a real HF channel behaviour is by removing the time and frequency restrictions of the Watterson model whilst keeping the same model structure. This approach has been followed in [2]. The underlying problem with the modifications of the basic model is the corresponding increase of the implementation complexity. Fortunately the technology of DSPs has evolved so much that it is possible now to face this increased complexity.

Aligned with the above mentioned, the present contribution describes a modified Watterson model to be implemented in a PC based platform equipped with only one general purpose DSP board. The emphasis is put on the implementation aspects of the platform more than in the likelihood of models to be brought to it. The following section provides a summary of the model as well as the ranges of the parameters which are allowed to be modified. The next section enters in details of functional and architectural aspects of the simulator platform. Although not presented here, it is believed to provide a system demonstration during the Conference.

\section{THEORETICAL MODEL}

\section{Background}

This contribution is focussed on an implementation approach of a possible modification of the Watterson model. The modification consists of allowing some of the model parameters, which are constant in the original model, to be slowly time varying according to the user requirements and under the control of a smoothing algorithm intented to force the change smoothly as it would probably occur in the real channel.

The Watterson model has been for a long time the paradigm for simulating the propagation effects on analog signals caused by the lonosphere. This model has many advantages which can be summarized on the fact that its complexity is low, and therefore real time implementations are easily reachable with present days technology, and it represents with a reasonable degree of accuracy the behaviour of the HF channel under some specific conditions. But this model is not exempted from drawbacks. In fact, it is a narrowband model and some of the new HF modems, although still transmitting in a narrowband channel, may exhibit frequency agility, thus making useless such kind of model. Even during the time interval when the modem is working at a given carrier frequency there are many propagation effects which can not be simulated by means of the Watterson model due to the fact that it is a WSSUS model and as it is know, the HF channel may follow this model for no more than a few minutes. In fig. 1 this model is represented. 


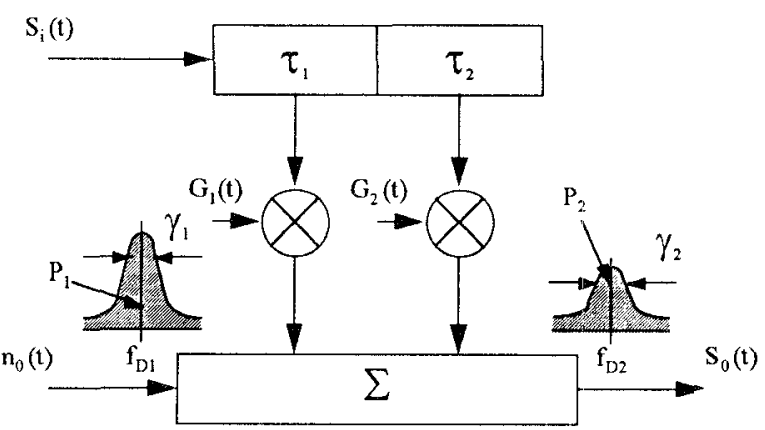

Figure 1: Basic Watterson model

\section{Modified Watterson model}

The Watterson model previously mentioned incorporates several defined statically parameters. These parameters are the following:

- Delay of each one of the paths.

- Frecuencial Dispersion of each one of the paths.

- Number of Paths to simulate.

- Doppler Displacement of each one of the paths.

- Power Relation between the paths.

- White noise level.

Through the present platform, it is possible to define for each parameter a variation margin, as well as a model and an predefined interval of variation. The predefined variation models are the following: Sinusoidal model, triangular model and gaussian model. The temporary intervals can be increased from $10 \mathrm{~ms}$ until $10.000 \mathrm{~s}$ and the values permitted for each parameter, in discreet jumps, are the following:

- Delay: 0 to $5.5 \mathrm{~ms}$

- Frecuencial dispersion: $0.11 \mathrm{~Hz}$ to $54.4 \mathrm{~Hz}$

- Numbers of paths to simulate: 4 with one magnetoionic component or 2 with two magnetoionics components.

- Doppler shift: $-100 \mathrm{~Hz}$ and $+100 \mathrm{~Hz}$

- Power relation between the paths: 0 to $40 \mathrm{~dB}$

- White Noise level: $+6 \mathrm{dBm}$ to $-40 \mathrm{dBm}$

Once it is established in the $P C$ the value of the parameters and its variation, with the execution of the program the parameters pass dynamically to the simulator DSP from the PC bus. Collects the new parameters and updates the simulator to the new values. The rate that the parameters pass from the PC program to the simulator, it is adjusted automatically by the algorithm of the PC, due to the fact that the variations will be smoothened.

\section{SIMULATOR DESCRIPTION}

The platform intented to implement this model consists of a DSP board based on the TMS 320030 and a control algorithrn running on the same PC hosting the above mentioned board. The control algorithm is entrusted to process the user requirements at platform programming time and to send the appropriate data and commands to the DSP at run time in order to fulfill such user requirements. The idea is simple because in a small time interval the DSP board can be seen as implementing a conventional Watterson model with a given combination of channel parameters, but in the following time interval one or more of these parameters will be changed and the DSP will be forced to implement a sligthly different model. The updating information and updating time patterns will be generated and managed by the control algorithm running in the $P C$ and data will be transferred to the DSP by DMA. The key point in this process is to avoid disruptions that in any way would appear in real HF operation.

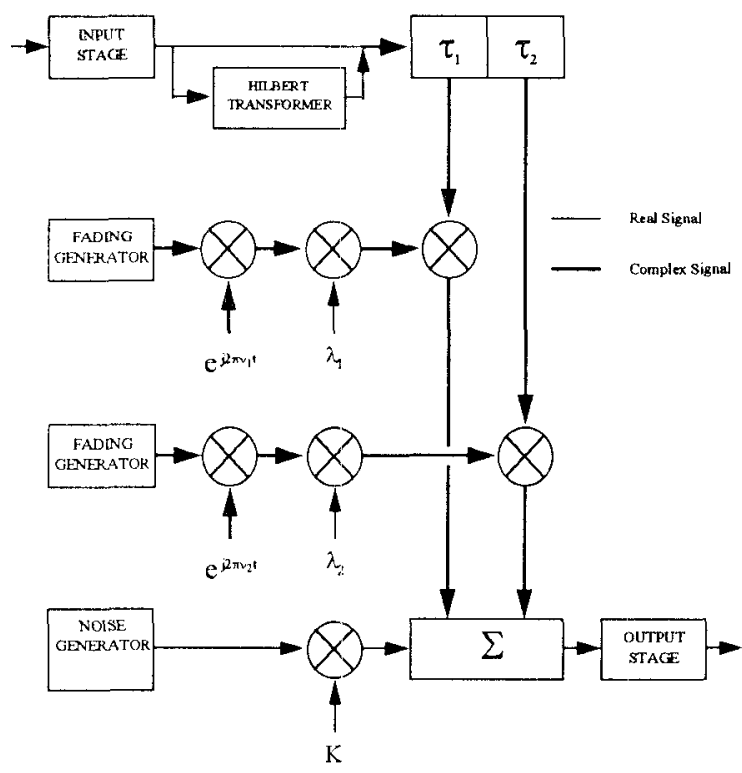

Figure 2: Simulator architecture

Figure 2 shows the general plan of the simulator. It is formed by an input stage, a Hilbert transformer to obtain the analytical signal, a delay line with four adjustable taps, four branches that provide each one of then the fading and the corresponding frequency shift for a simple path or for a magnetoionic component, an additive noise generator and an output stage. The $\lambda_{i}$ and $K$ factors that appear in the figure are the weighting 
factors of the fading and of the additive noise, respectively. The simulator has been implemented in the Texas Instruments EVM30 card of the DSP TMS $320 \mathrm{C} 30$, figure 3 , and all the modules have been accomplished in software, some in language $\mathrm{C}$, and other in assembly.

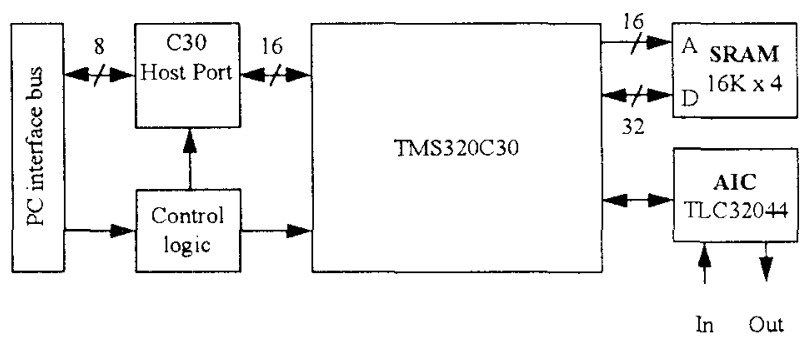

Figure 3:Texas Instruments EVM30 Card

The input stage, includes the input filter, the sampling and the analog-digital converter, is accomplished in the integrate circuit $\mathrm{AIC}$ TLC32044, programmed to carry out a $6400 \mathrm{~Hz}$ sampling rate, and a bandwidth of $300 \mathrm{~Hz}$ to 2.700 $\mathrm{Hz}$.

One of the main characteristic of the HF link is the multipath propagation of the transmitted signal. Such phenomenon causes that the signals that arrive to the receiver are formed each one of then by several components whose spread delays are scattered in a time interval that in some occasions can become of several miliseconds. To reproduce this effect, the simulator has a delay line with four adjustable taps that permits to simulate spread delays of up to $5.5 \mathrm{~ms}$.

The fading generation is the most complex task and at the same time more important of all those which are carried out during the simulation process. The simulator has four fading generators, one for each magnetoionic component that processes. Each one of them is formed by a gaussian random process generator and by a spectrum shape filter. The gaussian process generator is constituted by a uniform process generator and then applied the Walsh-Hadamard transform. The spectrum shape filter would have a gaussian frecuencial response. This has been accomplished through an order 3 Butterworth filter, whose frecuencial response coincides with a hypothetical gaussian filter in the point where the $3 \mathrm{~dB}$ fall is produced. As the frecuencial dispersion of the random processes can reach values approximately between $0,1 \mathrm{~Hz}$ and $50 \mathrm{~Hz}$, it is observed that the filter cutoff frequency is very small with respect to the employed sampling frequency $(6.400 \mathrm{~Hz})$.

This means that the filter will have a very narrowband that supposes serious problems to guarantee the stability of the filter. To solve this problem one sampling frequency reduction process has been adopted, through a decimation, before the filtering and finally an interpolation process.

A frecuency shift (Doppler) is produced as consequence of the variation with the time of the average height of the layers that compose the ionosphere. This effect is simulated multiplying each magnetoionic component by a exponencial complex signal. The frequency and the sign associated with this exponencial indicate the magnitude and the sense of the Doppler shift. The margin in this platform is going to be between -100 $\mathrm{Hz}$ and $100 \mathrm{~Hz}$.

The simulator permits the possibility of adding white gaussian noise to the transmitted signal. Such noise is generated in the same way that they are generated in the random processes that simulate the fading, with the exception of the fact that it is not necessary to configure its power spectrum profile. The noise levels can be between $+6 \mathrm{dBm}$ and $-40 \mathrm{dBm}$.

Finally the output stage include the digital-analog converter and the output filter. All this process is accomplished in the AIC TCL32044, programmed adequately.

\section{CONCLUSION}

A working prototype of the above described platform has shown that the ideas expressed in this contribution can be put into practice with the existing technology, In fact, the limitations on the ranges of the parameters are only disctated by the fact that we decided put only one DSP board of predefined characteristics. The work may be continued in two paralell lines. In one we would consider the remouval of technological constraits, by using a more powerfull platform. And in the other it would be necessary to try to get realistic variation models from HF propagation measurements.

\section{REFERENCES}

1. Watterson, C.C., Juroshek, J.R., and Bensema, 1970, "Experimental confirmation of an HF Channel Model", IEEEtrans. on communications, 18, $\underline{792-803}$

2. Van der Perre, L. and Van de Capelle, A., 1995, "Study of the HF-channel with the view of the use of wideband communication techniques", HF 95 nordic HF-conference, $\underline{1.6 .1-1.6 .9}$ 